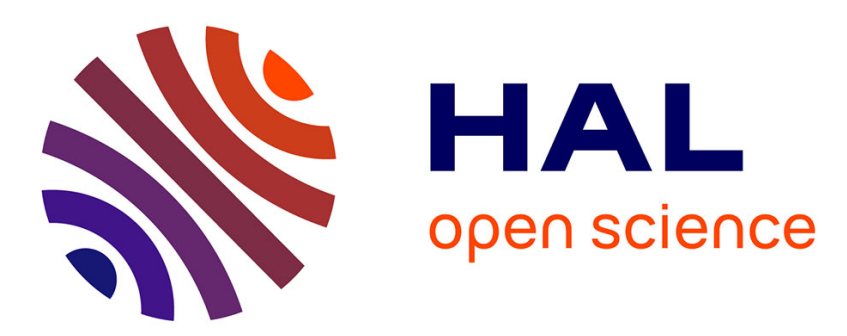

\title{
Towards resilient cyber-physical systems: The ADREAM project
}

\author{
Jean Arlat, Michel Diaz, Mohamed Kaâniche
}

\section{To cite this version:}

Jean Arlat, Michel Diaz, Mohamed Kaâniche. Towards resilient cyber-physical systems: The ADREAM project. 9th IEEE International Conference on Design \& Technology of Integrated Systems in Nanoscale Era (DTIS-2014), May 2014, Santorin, Greece. pp.1 - 5, 10.1109/DTIS.2014.6850642 . hal-01419019

\section{HAL Id: hal-01419019 \\ https://hal.science/hal-01419019}

Submitted on 18 Dec 2016

HAL is a multi-disciplinary open access archive for the deposit and dissemination of scientific research documents, whether they are published or not. The documents may come from teaching and research institutions in France or abroad, or from public or private research centers.
L'archive ouverte pluridisciplinaire HAL, est destinée au dépôt et à la diffusion de documents scientifiques de niveau recherche, publiés ou non, émanant des établissements d'enseignement et de recherche français ou étrangers, des laboratoires publics ou privés. 


\title{
Towards Resilient Cyber-Physical Systems: The ADREAM Project
}

\author{
Jean Arlat, Michel Diaz and Mohamed Kaâniche
}

LAAS-CNRS and Université de Toulouse, 7, Av. du Colonel Roche, BP 54200 - 31031 Toulouse Cedex 4 - France

\begin{abstract}
Advances recently witnessed in pervasive and ubiquitous information processing, driven by major breakthroughs in current cyber-physical systems (CPS) are paving the way towards a more hospitable and sustainable future based on a more efficient management of our environment: homes, work places, public areas, etc. Still, many advances have to be made to tackle several scientific challenges and to address related social, legal and ethical concerns attached to such highly dynamic networked service-oriented human-aware CPS. Towards this ends, LAAS-CNRS has launched a comprehensive and innovative multidisciplinary research program entitled ADREAM: Architectures for Dynamic Resilient Embedded Autonomous Mobile systems. This paper illustrates several of these challenges and briefly presents the research activities conducted as part of the ADREAM program, with a special highlight on dependability and resilience issues.
\end{abstract}

Keywords - cyber-physical systems; smart objects; ambient intelligence; Internet of things; resilience.

\section{CONTEXT AND MOTIVATION}

Advances recently witnessed and increasingly emerging in computerized embedded systems - pervasive and ubiquitous deployment in the physical environment and in many instances in close interaction with humans - have led to the paradigm of cyber-physical systems (CPS). As it is put forward in [1], this term refers to the tight interaction and coordination between computational and physical resources.

The main features concern the wide and pervasive diffusion of increasingly integrated and miniaturized smart nodes (including at the micro- and nano-scale for some of them) that provide (fully ${ }^{1}$ or partly) the following advanced capabilities: data collection (sensors), communication (openness and interaction), information processing (analysis, optimization and decision), and action on the environment — in a wide sense - (actuators). Those capabilities fully characterize the concepts of Ambient Intelligence and Internet of Things.

1 Autonomous systems (e.g., robots) are a good example of the materialization of the monitoring-decision-control loops necessary to ensure their operation in an unknown dynamic environment in which they are embedded.
Beyond the properties of cross-interacting together and of impacting their environment, another important key feature of CPS refers to interactions with humans. Indeed, these interactions span well beyond the human beings interfaced with embedded systems (e.g., the operators in charge of steering and checking of such systems) and apply to the general public and also to a wide range of the society. As notable examples, one can think of:

- the future autonomous transportation systems (e.g., in avionics or automotive domains);

- the manned space flights (orbital rendez-vous, future interplanetary missions);

- the "silver economy" (monitoring and assistance to the elderly or handicapped people), as well as the medical supervision;

- the operation and monitoring of infrastructures (resource supply, transportation systems, communication networks);

- the development of a sustainable and efficient agriculture (control of the inputs, monitoring of the crop, manure processing);

- added-value information and services (friendly situation awareness in public urban spaces);

- the cockpit/factory of the future (new generation of robots to check/assist the pilots/workers in their tasks);

- search and rescue operations, exploration, firefighting and crisis management (deployment of aerial and ground robot fleets, sometimes in connection with human actors).

Indeed, CPS are paving the way towards a more hospitable and sustainable future featuring a more efficient management of our environment: homes, offices, factories, public areas, etc. [2].

The requirements for these systems are faster response time (e.g., autonomous collision avoidance systems), more precise operation (nano-tolerance manufacturing or robot-assisted surgery), autonomous and large scale deployment (access to dangerous locations), distributed coordination (automated traffic control), highly efficient (zero-net energy buildings, smart resilient and secure heterogeneous energy grids), augmented human capabilities, and enhanced societal wellbeing (e.g., assistive technologies and ubiquitous healthcare monitoring and delivery). 
Beyond the public and societal benefits in everyday life that are often put forward, such innovative technologies based on smart objects (sensors, actuators) will have a strong economical impact on the enterprises and the factories of the future by supporting the emergence of more digitalized, interconnected and agile production facilities. Several complementary priorities are at stake in that domain ${ }^{2}$ that encompass the joint development of more advanced manufacturing processes including adaptive, customer-focused and human-centered smart manufacturing systems. This is reflected for example by the emerging concept of co-worker robots and the increasing trend toward deploying sensors and Internet technologies in industrial factories.

This vision and the benefits promised are definitely appealing. However, the requirements for the CPS of the future will far exceed those of today systems in terms of functionality, usability, adaptability, autonomy, timeliness and resilience to accidental and malicious threats, as well as to dynamic changes of the user behaviors and of the environments. Actually, for this to become a reality, many advances have still to be made to tackle several scientific challenges and to address related social, legal and ethical concerns attached to such highly dynamic networked service-oriented human-aware CPS.

LAAS-CNRS has promoted, as early as the mid 2000's, studies on open autonomous and distributed embedded systems, thus anticipating the emerging CPS. Towards this end, it was decided to initiate a multidisciplinary transverse research project, entitled ADREAM: Architectures for Dynamic Resilient Embedded Autonomous Mobile systems.

In the sequel, we first briefly give the rationale for the ADREAM project. Section III summarizes the main features of the research program. Section IV describes the experimentation platform that supports most of these activities. Finally, Section V draws some conclusions and identifies some perspectives for future work.

\section{THE RATIONALE FOR ADREAM}

The progress needed to tackle the challenges at stake definitely requires joint efforts in a multidisciplinary approach that encompasses (at least) the deployment and usage of low power sensors, ad hoc communication protocols, mobile and autonomous agents (including robots), reconfigurable and adaptive computing platforms, sophisticated optimization and robust control, advanced management of electrical energy (harvesting, production, conversion, storage, distribution, etc.).

All of this relies on the achievement of high levels of quality of service (QoS) and dependability, especially with respect to a very dynamic context and environment (hence the need for resilience [3]).

The massive deployment of sensors and the collection of large sets of data providing detailed information about user

\footnotetext{
2 http://www.effra.eu.
}

behaviors, location and personal data call for the development of innovative solutions that preserve privacy without constraining too much the usability and the functionality of the systems.

LAAS-CNRS has identified, already in 2006, the interest of distributed intelligence together with the trend towards cyberphysical systems by promoting the emergence of open autonomous embedded systems, first as a relevant and challenging frontier to target, and second also as a mean to build up synergies among the activities carried out by several of its research teams. Indeed, the research conducted spanning Critical Computing, Networks \& Communication, Robotics, Automatic Control, Optimization \& Diagnosis and Micro \& Nano Systems - largely covers the pertinent topics identified in the previous section. It was thus decided to launch the transverse project entitled ADREAM: Architectures for Dynamic Resilient Embedded Autonomous Mobile systems. The research agenda aims to investigate and develop core technologies, methodologies and components that will enable the successful design, assessment and deployment of dependable CPS that directly interact with humans, and that can be used by a large number of applications.

\section{Accordingly, ADREAM [4] is both:}

- A research program that encompasses a set of coordinated research actions addressing several of the ambitious and interdisciplinary challenges posed by CPS, and,

- An experimentation platform that supports the development of most of the ADREAM research actions. It is a new building, fully equipped with sensors, robots, networks, embedded devices, and photovoltaic electricity production facilities. This platform itself is also the subject of specific research actions involving the set of instrumentation available, as well as the people residing in the building.

In the sequel, we describe these two facets.

\section{The RESEARCH PROGRAM}

ADREAM develops research on methods, devices, services and tools, and also implement experiments for assessing new cyber-physical systems. Eight main topics are explored to address the technologies and methodologies for the efficient development and operation of CPS.

Six are very much related to the design of components, architectures and services attached to the deployment of CPS - micro and nano sensors, autonomic ubiquitous computing systems, localization, navigation, robotics and mobility, optimization and control, energy systems, and security and privacy.

The two others, namely, system co-simulation and covalidation environments, and formal development of adaptive mobile systems, are geared towards the important issues of the assessment of non-functional attributes (especially, QoS, dependability, security and resilience) during the related complex design phases. 
It is noteworthy that (at least) half of them are directly connected to dependability and resilience issues. We briefly describe them hereafter.

\section{A. Autonomic Ubiquitous Computing Systems}

This action is dedicated to the design and development of methods, hardware and software mechanisms and tools for providing self-configuration, self-protection and selfadaptation, and more specifically [5], addressing:

- complex system monitoring, data gathering and clustering;

- context analysis, for adaptive diagnosis and prognosis, and decision making;

- operational dynamic reconfiguration of the distributed smart objects, the communication stacks and protocols, the resilience mechanisms and the services offered at the application layer.

The aim is to develop the components and services (hardware and software) as well as the requested deployment rules for a fully distributed infrastructure, including the selection of the most adapted communication protocols.

It encompasses all the control phases defining the management cycle for providing the properties of self-protection and selfadaptation of Machine-to-Machine (M2M) systems [6].

Concerning dependability issues, the challenges addressed include:

- the resilience of ubiquitous systems;

- the adaptive quality of service and the intelligent control of autonomic communications;

- the unsupervised detection of network traffic anomalies.

\section{B. Security and Privacy}

CPS rely on a large set of interconnected and heterogeneous objects (from very simple sensor devices to mobile autonomous systems). Security and privacy are important issues as the applications deployed are often vulnerable to attacks.

Because of strong interactions with the humans, the collected, processed, sent or stored data (personal, commercial, home, medical, localization, etc.) can be very sensitive in many applications of CPS. This is addressed by developing the two following types of investigations:

- vulnerability analysis and protection of largely deployed home-network equipment connected to the Internet.

- protection of personal data related to localization-based applications.

In the first case, studies are currently carried out for analyzing and comparing the security mechanisms implemented in French ADSL boxes.

In the second case, the aim is to design innovative security technologies that give people control over their personal information and prevent applications and devices from disclosing such information without the consent of their users. Indeed, collection of individuals' mobility data on a daily basis is one of the major threats against privacy. For that, a specific PRivacy-preserving 1Ocation Proof protocol, has been proposed [7].

\section{Formal Development of Adaptive Mobile Systems}

Mobility and adaptive features that characterize the CPS considered in ADREAM pose some specific difficulties for the usual verification and evaluation approaches [8]

This will be tackled in a comprehensive way via complementary research actions:

- formal development and model-checking, including issues such as automatic synthesis of controllers and code generation;

- software testing addressing the challenges raised by mobility and autonomy properties in uncertain environments [9];

- performance and resilience evaluation for optimized decision, scheduling and planning, both at design-time and at run-time.

The results of these on-going investigations are expected to provide two types of contributions:

- on one hand, the development and validation of the different phases of the design of critical embedded systems, especially when considering tight timing constraints and dynamic scheduling;

- on the other hand, the application, evaluation and consolidation of these methods used, developed or extended in the context of ADREAM to address increasingly complex interconnected systems, especially for what concerns the scaling up of verification methods and techniques (processing speed-up, memory usage, etc.) [10].

\section{Co-simulation and Co-validation Environments}

Powerful co-simulation and co-validation techniques are needed to build and analyze complex interdisciplinary multitechnology systems, such as the ADREAM platform [11].

As validation means comparing models to real world behaviors, the research work carried out in this context is conducted in close link with the results obtained via the previous formal approach (Section C), either for producing efficient test sequences, or for validating or strengthening the simulation results.

The research conducted addresses the following items:

- definition of a global simulation framework, including various sets of heterogeneous and hybrid models at different system abstraction layers;

- identification of validation metrics and of their formal representations;

- selection of specification languages suited for supporting the generation of test data;

- development of effective simulation methods and tools. 


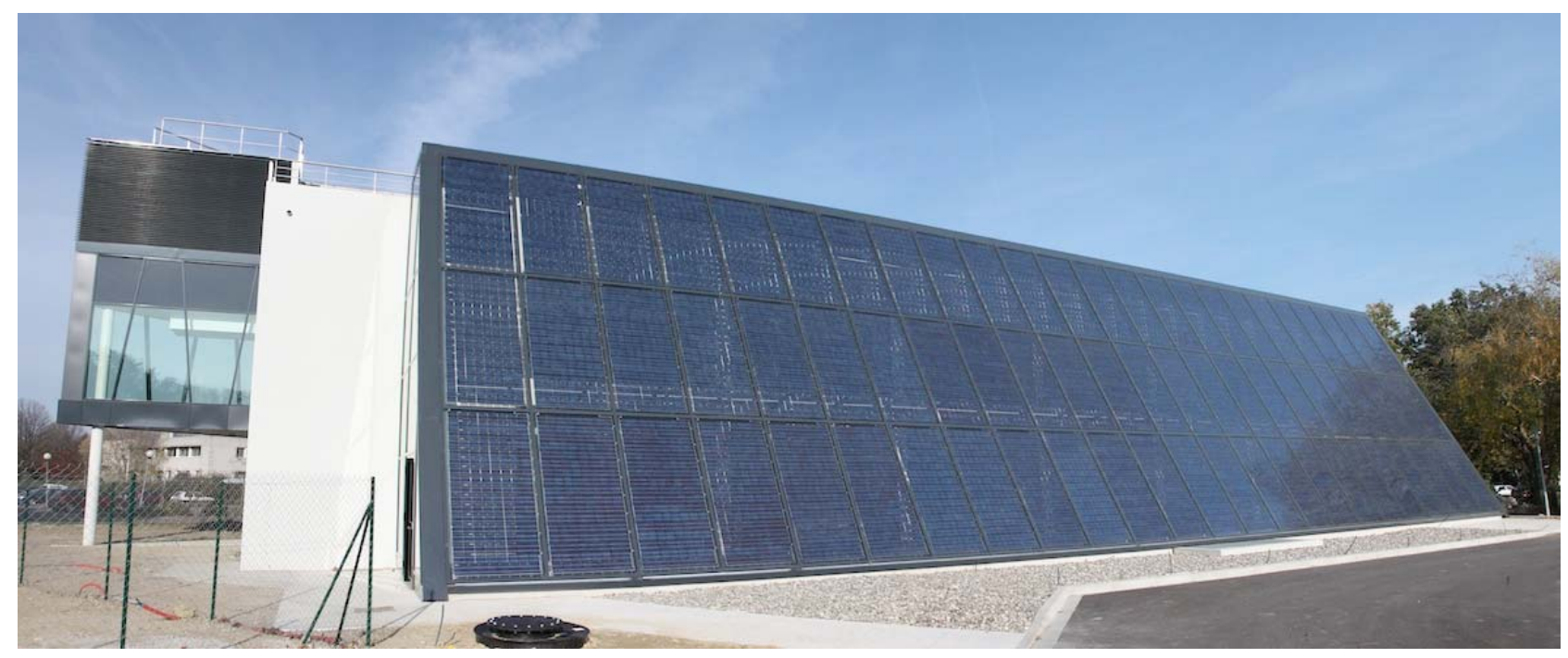

Fig. 1. The Georges Giralt Building: The Photovoltaic Main Windowed Façade

\section{THE EXPERIMENTATION PLATFORM}

The ADREAM project relies heavily on an experimentation platform that is materialized by an innovative building ${ }^{3}$. The building provides an advanced environment for the design and the validation of ubiquitous, autonomous, resilient and energyaware management systems by using pervasive computing and autonomous intelligence. It integrates a large set of different devices into a comprehensive integrated framework featuring sensors, actuators, networks, robots, etc. The building is powered by a dedicated micro grid managing the production of $100 \mathrm{KWc}$ from solar energy via a set of photovoltaic (PV) panels of more than $700 \mathrm{~m}^{2}$ (including bi-glass and tri-glass modules on the windowed façade).

The platform incorporates many complementary knowledge and skills from the electronics, energy, decision, robotics and critical computing systems research areas. As such, it provides a privileged framework to demonstrate, by designing and deploying a real complex system, the innovative facets from the research conducted on these domains by LAAS-CNRS and its partners.

The main features of the platform, unique in Europe, today include:

- a PV electrical production system, with inverters, energy storage facilities, and lab benches connected to the PV panels;

- a "décor" apartment for experimenting M2M systems, monitoring people behaviors, and devising companion robots for decision planning and interaction with the humans;

- three autonomous robots, cameras and vision sensors, presence sensors, sensors for measuring the electricity

3 The "Georges Giralt" building. G. Giralt was one of the two founding Directors of laboratory in 1968. He has developed seminal research on power electronics and launched the research activities in robotics at LAAS-CNRS. production and consumption of the main building subsystems, environmental sensors (weather, etc.).

The research program and the platform are open to collaborations that we would be happy to discuss and set up, in order to address joint research, in particular on: optimal production and consumption of power energy, environment perception and interpretation, robot-human cooperation, design of sensor networks, protocols and services for machineto-machine communications, all being able to fulfill stringent dependability, security and privacy constraints.

\section{CONCLUDING REMARKS}

The ADREAM project provides a rather unique framework and environment in which several of the challenges attached to cyber-physical systems (CPS) are addressed on a really comprehensive basis. This is made possible thanks to the wide spectrum of relevant research activities and competences that are developed at LAAS-CNRS.

Interdisciplinary approaches become the main focus for the research being conducted. Examples include:

- the development of various types of sensors (e.g., foot/wrist fall detectors), advanced communication devices (highly miniaturized, consumption-free transducers) and low energy, long range communication networks (including M2M layered protocols).

- the command-control of a fleet of drones, encompassing complex issues such as: coordinated planning and actions, distributed robust control, algorithm optimization.

- a distributed control strategy coordinating the collection, and the processing of the various data produced within the instrumented platform for optimizing the control and regulation of the building (temperature, lightning, etc.).

It is also worth pointing that we are ready to enlarge our cooperation with other labs and disciplines. Actually, the 


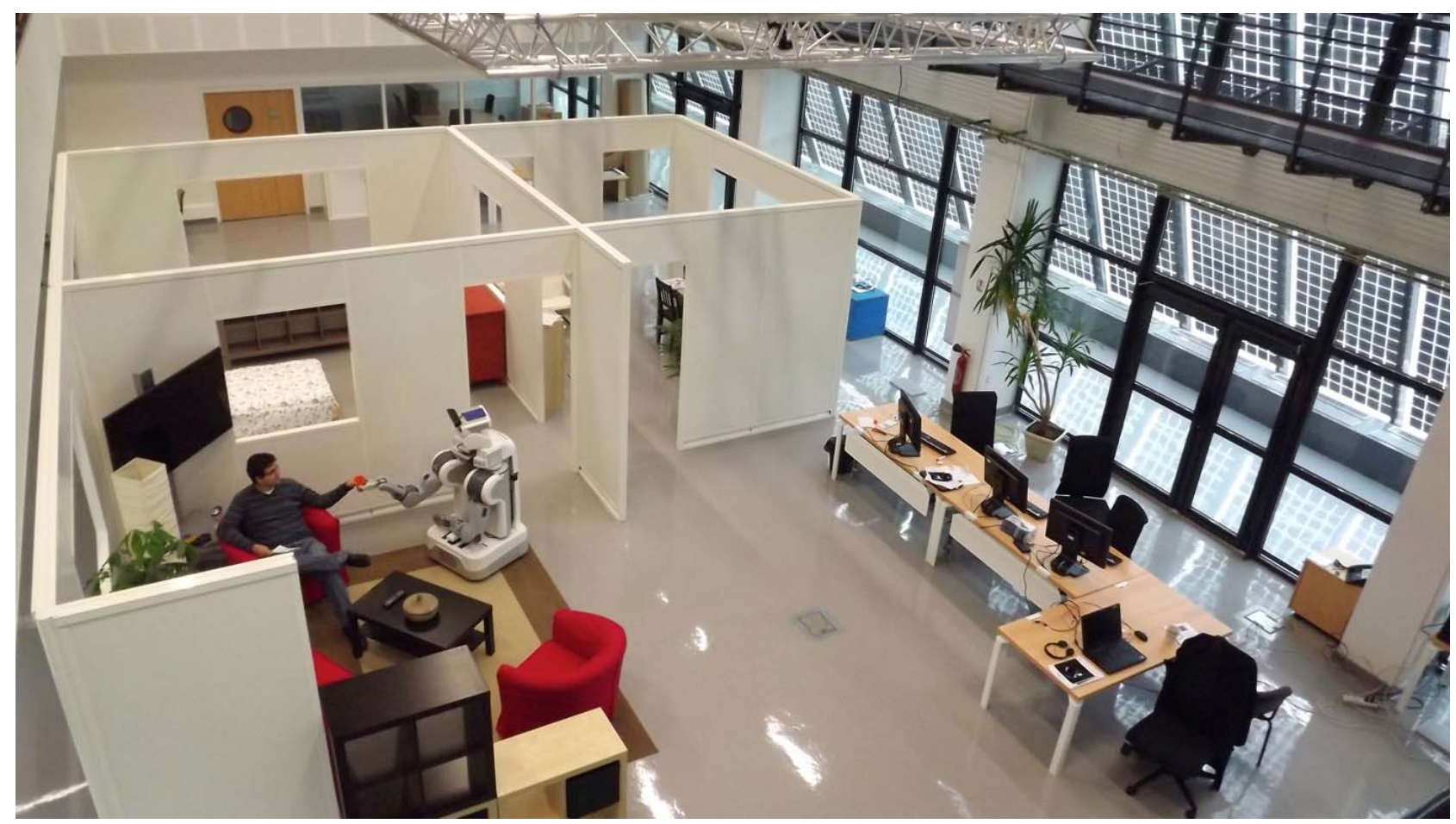

Fig. 2. The Cyber-Physical Systems Experimentation platform

energy-related platform from the building is already a shared facility. The results from the collection and analysis of the sensor data of the building will benefit the NeOCampus project recently initiated on the University campus.

Another target for cross-fertilizing investigations include the joint consideration of the technical, regulatory, economic and social constraints that are underlying the deployment and acceptance of CPS. This joint effort, aimed at investigating a multidisciplinary approach for designing CPS, has received the support from the STAE Foundation.

\section{ACKNOWLEDGMENT}

We thank all the people at LAAS and CNRS who have contributed to the definition and development of the ADREAM project. We also gratefully acknowledge the funding received as part of the "CPER 2007-2013 Program", from the European Union via the FEDER, the French State, the Midi-Pyrénées Région, the Urban Community Toulouse Métropole, CNRS, LAAS, and the Club des Affiliés du LAAS-CNRS.

\section{REFERENCES}

[1] E.A. Lee, "Cyber Physical Systems: Design Challenges", Proc. 11th IEEE Object Oriented Real-Time Distributed Computing (ISORC), (Orlando, FL, USA), pp. 363-369, 2008.

[2] R. Rajkumar, I. Lee, L. Sha, J. Stankovic, "Cyber-Physical Systems: The Next Computing Revolution", Proc. 47th ACM/IEEE Design Automation Conf. (DAC), pp.731-736, 2010.

[3] J.-C. Laprie, "From Dependability to Resilience", Proc. 38th Annual IEEE/IFIP Int. Conf. Dependable Systems and Networks (DSN-2008) Fast Abstracts, (Anchorage, AK, USA), 2008.
[4] ADREAM: Vers la Conception et l'Evaluation des Systèmes Cyberphysiques - Programme Scientifique, LAAS-CNRS Rep., Dec. 2012, 98p. (In French) - An abridged version is also available as "ADREAM: The Research Program and the Experimentation Platform", LAAS-CNRS Rep., Dec. 2012, 12p. — www.laas.fr/ADREAM.

[5] T. Braun, M. Diaz, J.E. Gabeiras, T. Staub, Eds, "End-to-end Quality of Service Over Heterogeneous Networks”, Springer, 2008.

[6] M. Ben Alaya, S. Matoussi, T. Monteil, K. Drira, "Autonomic Computing System for Self-management of Machine-to-Machine Networks", Proc. ACM Int. Workshop on Self-aware Internet of Things, series (Self-IoT '12), (San Jose, CA, USA), pp. 25-30, 2012.

[7] S. Gambs, M.-O. Killijian, M. Roy, M. Traoré, "Locanyms: Towards Privacy-Preserving Location-Based", Proc. 1st European Workshop on AppRoaches to MObiquiTous Resilience, (ARMOR, 2012), (Sibiu: Romania), http://hal.archives-ouvertes.fr/hal-00699742.

[8] A. Bondavalli, O. Hamouda, M. Kaâniche et al., "The HIDENETS Holistic Approach for the Analysis of Large Critical Mobile Systems", IEEE Trans. on Mobile Computing, Vol. 10, no 6, pp. 783-796, 2011

[9] P. André, N. Rivière, H. Weaselynck, "GraphSeq Revisited: More Efficient Search for Patterns in Mobility Traces", Proc. European Workshop on Dependable Computing (EWDC 2013), (Coimbra, Portugal), Springer LNCS 7869, 2013.

[10] R.T Saad, S. Dal Zilio, B. Berthomieu, "An Experiment on Parallel Model Checking of a ctl Fragment", Proc. 10th Int. Symp. Automated Technology for Verification and Analysis (ATVA 2012), (Thiruvananthapuram, India), pp. 284-299, 2012.

[11] D. Foures, V. Albert, A. Nketsa, "Simulation Validation Using the Compatibility between Simulation Model and Experimental Framework", Proc. Summer Simulation Multi-Conference (SummerSim), (Toronto, Canada), pp. 720-726, 2013. 